\title{
Interpretation of motion analysis of laparoscopic instruments based on principal component analysis in box trainer settings
}

\author{
Ignacio Oropesa · Fernando Pérez Escamirosa · Juan A. Sánchez-Margallo · Silvia Enciso · \\ Borja Rodríguez-Vila A Arturo Minor Martínez • Francisco M. Sánchez-Margallo • Enrique J. Gómez • \\ Patricia Sánchez-González
}

\begin{abstract}
Background Motion analysis parameters (MAPs) have been extensively validated for assessment of minimally invasive surgical skills. However, there are discrepancies on how specific MAPs, tasks, and skills match with each other, reflecting that motion analysis cannot be generalized independently of the learning outcomes of a task. Additionally, there is a lack of knowledge on the meaning of motion analysis in terms of surgical skills, making difficult the provision of meaningful, didactic feedback. In this study, new higher significance MAPs (HSMAPs) are proposed, validated, and discussed for the assessment of technical skills in box trainers, based on principal component analysis (PCA).

Methods Motion analysis data were collected from 25 volunteers performing three box trainer tasks (peg grasping/PG, pattern cutting/PC, knot suturing/KS) using the EVA tracking system. PCA was applied on 10 MAPs for each task and hand. Principal components were trimmed to those accounting for an explained variance $>80 \%$ to define the HSMAPs. Individual contributions of MAPs to HSMAPs were obtained by loading analysis and varimax rotation. Construct validity of the new HSMAPs was carried out at two levels of experience based on number of surgeries.

Results Three new HSMAPs per hand were defined for PG and PC tasks, and two per hand for KS task. PG presented validity for HSMAPs related to insecurity and economy of space. PC showed validity for HSMAPs related to cutting efficacy, peripheral unawareness, and confidence. Finally, KS presented validity for HSMAPs related with economy of space and knotting security.

Conclusions PCA-defined HSMAPs can be used for technical skills' assessment. Construct validation and expert knowledge can be combined to infer how competences are acquired in box trainer tasks. These findings can be exploited to provide residents with meaningful feedback on performance. Future works will compare the new HSMAPs with valid scoring systems such as GOALS.
\end{abstract}

Keywords Box trainer $\cdot$ Motion analysis $\cdot$ EVA tracking system $\cdot$ Principal component analysis $\cdot$ HSMAP

Technology-enhanced learning has changed the way in which training and assessment of minimally invasive surgery (MIS) technical skills are performed, especially outside the operating room (OR). Box trainers enhanced with instrument tracking capabilities, virtual reality (VR) simulators or serious games are nowadays extensively used in laboratory settings, allowing residents deliberate practice of skills in patient-free, safe environments. However, an important aspect remains on how to measure technical performance, and more significantly, how measured skills transfer into the OR $[1,2]$.

Motion analysis has been proved to be an effective and valid assessment tool for technical skills in different fields of MIS. Metrics such as path length, average speed or economy of area have been proposed to discriminate between surgeons with different experiences (number of surgeries performed) and expertises (academic/professional level). Moreover, thanks to the popularization of commercial devices such as Leap Motion [3] or the development of video-based tracking technologies such as the EVA tracking system [4] or the EndoViS training system [5], the costs of incorporating laparoscopic instrument tracking into box trainers are constantly 
being reduced, making these assessment tools widespread in hospitals and training centers.

Construct validation of motion metrics in an assessment system is vital in determining its predictive capabilities of skills' transfer into the OR [6]. However, validity of motion analysis parameters (MAPs) varies depending on the skills assessed in a surgical task performed in a box trainer or VR simulator $[2,7]$. Furthermore, it is a well-established fact that one MAP alone does not provide adequate measures on performance, and at least two outcome measures are required [8]. Moreover, individual metrics in themselves do not provide meaningful information that can be translated into proper feedback to the resident (e.g., telling a resident that his/her obtained path length is of $1.5 \mathrm{~m}$ does not in itself have any didactic value) [9]. These issues raise the question on how best to combine MAPs for effective assessment, and whether said combinations may provide insight on the interpretability of results.

Interpretability is also reflected by the fact that it is still unknown how specific scores obtained for different MAPs in a box trainer translate into real performance in the OR. Despite studies reporting validity of individual metrics, there is no evidence on how these can be combined or related to higher-order metrics such as tissue handling or procedural efficiency. Several studies have studied the correlation between procedural scoring scales such as the Global Operative Assessment of Laparoscopic Skills (GOALS) [10] with the use of MAPs. In [1], authors showed that MAPs (time, path length and, in a lesser degree, economy of area and economy of volume) obtained in a box trainer suturing task correlated with the scores granted by two independent surgeons using a specific suturing checklist. The study, however, was limited to this particular skill, and thus did not broaden its scope to other important abilities such as depth perception, grasping, or cutting. Chang et al. [11] established a correlation of 0.93 between a global rating scale and the MAPs obtained in VR/training arthroscopy simulators. However, they did not examine the relation and meaning of the different MAPs with respect to the quality of performance.

In this study, we propose and validate new composite higher significance MAPs (HSMAPs) built from individual MAPs using principal component analysis (PCA). PCA is a common technique used in machine learning applications as a tool to detect redundancies between parameters and reduce data complexity. It has been previously used in the literature for automatic assessment of surgical skills in [12-15]. However, in these studies, no indication is given on the composition of the principal components in terms of the original metrics. The main goal of this work ties in with that presented by the authors in [1]: to determine if MAPs can be combined into higher significance assessment parameters able to provide a meaningful interpretation of assessment scores which could be later translated into comprehensive, constructive feedback for the surgical resident.

\section{Materials and methods}

The study is structured in four different phases: (1) data collection; (2) MAP exploratory analysis; (3) PCA for the definition of HSMAPs; and (4) HSMAPs construct validation. In the first phase, motion data from two laparoscopic instruments are collected in a box trainer setting using the EVA tracking system [4]. In the second phase, individual MAPs' correlation and construct validity are analyzed as a first step towards identifying the relevance of applying PCA, as well as to infer which motion elements are determining of surgical prowess. Phase three performs the actual PCA analysis and establishes the criteria for the definition of HSMAPs, while phase four performs construct validation on them. The following sub-sections will provide a detailed description of each phase.

\section{Data collection}

Data collection was carried out at the facilities of the Jesús Usón Minimally Invasive Surgery Centre (JUMISC, Cáceres, Spain). Volunteers were sought amongst residents partaking in courses or belonging to the teaching staff. Prior to the experiment, all participants were asked to fill a short questionnaire including demographic data and laparoscopic experience. Using these data, two different cut-off thresholds for surgical competence were investigated, based on previous laparoscopic experience. Thus, volunteers were categorized as belonging or not to the following study groups: (a) surgeons with $>10$ laparoscopic procedures performed (Ex10 vs non-Ex10); and (b) surgeons with $>50$ laparoscopic procedures performed (Ex50 vs non-Ex 50).

All participants were asked to carry out three laparoscopic tasks (shown in Fig. 1) in a SIMULAP® box trainer [16]:

- Peg grasping (PG) [4]: This task consists on grasping and placing spherical objects (chickpeas) on corresponding sequential holes. The scenario includes blocks at different heights, orientations, and with occlusion zones. This task is performed with the dominant hand and requires hand-eye coordination, spatial perception, and grasping skills.

- Pattern cutting (PC) [5]: This task requires cutting a $4.5-\mathrm{cm}$ circular pattern on a piece of white latex glove stretched in a plastic base. Using the laparoscopic scissors with their dominant hand, and with the support of a Maryland dissector in the non-dominant hand, participants must cut the drawn circle as close as possible, 

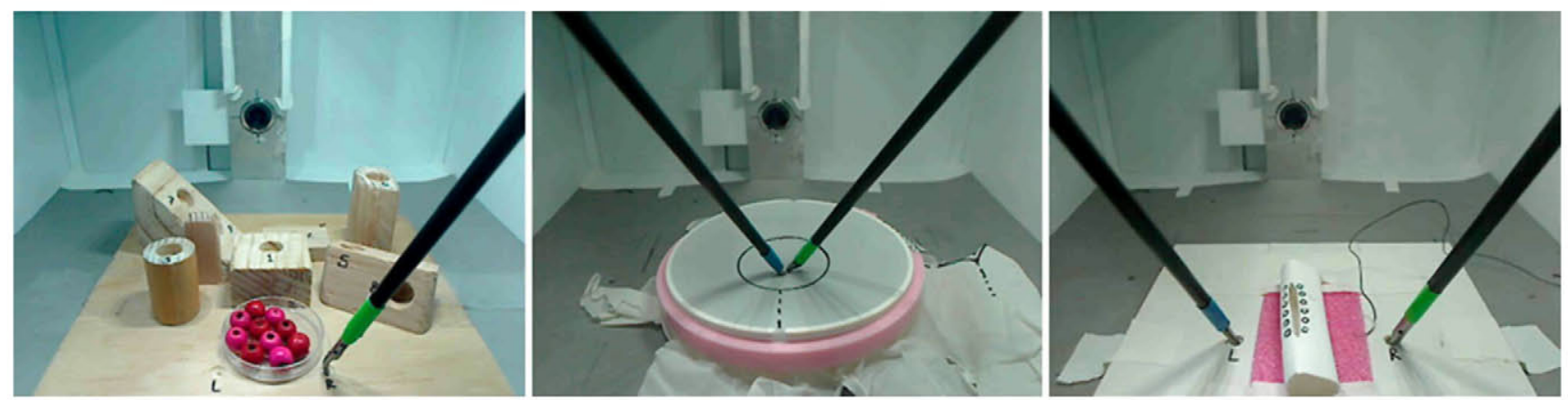

Fig. 1 Box trainer tasks. Left: peg grasping (PG). Center: pattern cutting (PC). Right: knot suturing (KS). (Color figure online)

starting and ending in the same position. The task ends when the circle is completely cut out and separated from the glove. This is a bimanual task that requires skills at cutting, grasping, bimanual dexterity, and hand-eye coordination.

- Intracorporeal knot suturing (KS) [5]: For this task, surgeons use a laparoscopic needle holder in their dominant hand and a Maryland dissector in their non-dominant hand. The task consists on grasping the needle with the laparoscopic needle holder, puncturing and knotting a 12-cm-long suture through two predefined points in a longitudinal slit Penrose drain. Next, a 2-0 silk suture is tied using an intracorporeal knot technique. This task requires skills at needle manipulation, management of silk suture, knot tying, bimanual dexterity, and hand-eye coordination.

For all tasks, the initial and ending positions of the instruments were predefined by two drilled holes on the task supporting board. All tasks were placed inside the SIMULAP® in identical positions. Camera was left fixed and in the same position for all participants. While most participants had previous experience with box trainers/VR simulators, none of them had ever encountered the specific versions of the tasks employed for this study. Thus, before starting, each participant was briefly instructed on the goals and steps of the task. No guideline on the strategy to follow in order to complete the task was given. Only one trial per task was allowed, and no time limit was imposed to complete them.

Tracking of laparoscopic instruments was performed using the EVA tracking system. EVA is a computer visionbased tracking system that obtains the instrument tip position based solely on the intrinsic camera parameters and the geometrical properties of the instruments, without the need of external sensors. First prototype of EVA was previously validated for motion analysis of MIS skills in box trainer settings [4]. The EVA tracking system is currently implemented in $\mathrm{C}++$, using OpenCV libraries to handle all image and video processing functionalities. EVA can track two laparoscopic instruments on the screen, provided that they each incorporate two different color markers (green and blue) near their tip. Moreover, tracking can either be performed in real time or a posteriori using video recordings of the task. In this instance, the latter method was used.

Tracking data were used to calculate ten different MAPs (Table 1). All MAP definitions were taken from previous

Table 1 Selection of MAPs and definition

\begin{tabular}{|c|c|}
\hline MAP & Definition \\
\hline Time & Total time to perform a task (s) \\
\hline Path length & Total path covered by the instrument within the setting (m) \\
\hline Depth & Total path length traveled in the instrument 's axis direction (m) \\
\hline Average speed & Rate of change of the instrument's position within the setting $(\mathrm{m} / \mathrm{s})$ \\
\hline Average acceleration & Rate of change of the instrument's velocity within the setting $\left(\mathrm{m} / \mathrm{s}^{2}\right)$ \\
\hline Motion smoothness & Abrupt changes in acceleration resulting in jerky movements of the instrument $\left(\mathrm{m} / \mathrm{s}^{3}\right)$ \\
\hline Bimanual dexterity & $\begin{array}{l}\text { Speed coordination (measured as correlation) between instruments controlled by dominant (D) and non-dominant (ND) } \\
\text { hands }\end{array}$ \\
\hline Idle time & Percentage of time where the instrument is considered to be still (speed $<5 \mathrm{~mm} / \mathrm{s})(\%)$ \\
\hline Economy of area & Relationship between maximum surface area (at camera plane) occupied by the instrument and total path length (-) \\
\hline Economy of volume & Relationship between maximum volume occupied by the instrument in the setting and total path length (-) \\
\hline
\end{tabular}


studies [4], with the exception of bimanual dexterity, introduced by Hofstad et al. [6]. Precise formulae for their calculation can be found in said studies. All MAPs were calculated using MATLAB® R2016b (Mathworks, NA, USA).

\section{MAP exploratory analysis}

Similarly to [15], a previous study on the correlation of MAPs was carried out using Pearson's correlation $(p>0.05)$ to look for linear relationships in the data, and see whether the use of PCA was justified. Strong correlations were defined by $\rho>0.7$. Mild correlations were defined by $0.4<\rho<0.7$. In order to validate the cut-off thresholds used in this study, a descriptive analysis of individual MAPs was performed prior to applying PCA. Since preliminary Shapiro-Wilk tests showed that not all data conformed to normal and/or symmetrical distributions, non-parametric Mann-Whitney test was employed to look for statistical significant differences between the medians of study groups for all variables $(p<0.05)$. All tests were conducted using SPSS v19 (IBM Corp, NY, USA).

\section{PCA for definition of HSMAPs}

PCA exposes the similarities and differences between features in a dataset, allowing its transformation based on intrinsic patterns. Principal components are extracted as linear combinations of the original observations (i.e., the MAPs), calculating the eigenvectors and eigenvalues of the covariance of the dataset. Eigenvectors provide the directions of maximum variance (or inertia) of the data, thus describing the existence of patterns within its structure. The first eigenvector reflects the direction of maximum amount of variance. The second component must necessarily be orthogonal to the first, and have the largest possible inertia, and so forth. Eigenvalues, on the other hand, reflect the weight of each eigenvector (i.e., the amount of variance in that direction). Geometrically, this can be seen as the projections of the observations onto the principal component/ eigenvector space [17].

The cumulative variance explained (CVE) reflects the total amount of variance/inertia given by the first $n$ components. The CVE is used as a criterion for trimming the dimensionality of the principal components [13-15]. If one is to transform the original dataset of observations and map it against the coordinate set defined by the eigenvectors, it is possible to first crop those components that do not meet a specific stopping condition (e.g., eigenvalues $>1$ ). Remaining components are known as the principal components, and are said to account for most of the variance in the dataset. Thus, by performing PCA, redundant features are identified and the dimensionality of the feature space is reduced.
An interesting application of PCA is that it can be employed to simplify and interpret the meaning of a dataset. This can be done by analyzing the contribution of the original variables to the principal components. Contributions can be defined as the ratio $(\%)$ of the squared covariance values (or, to use a specific terminology, loadings) between a variable and the component, and the total sum of squared loadings. The greater the ratio, the greater its contribution to the component.

Interpretation of components in terms of contributions can be complex, depending on the final structure taken by the eigenvectors. In order to achieve simpler structures, post-processing of the loadings' matrix can be performed in terms of rotations. One of the most commonly used rotations is the varimax, which transforms components in such way that the variance of individual squared loadings is maximized. This leads to a result where each original variable is associated with one or few components, and reciprocally each component represents only a small number of variables [17].

In this study, PCA is used for the definition of HSMAPs. Principal components were extracted for each task and hand (dominant and non-dominant) based on MAPs obtained by participants. These components define each of the HSMAPs. In each case, the stopping condition for defining components was set to an explained variance $>80 \%$. Prior to performing the analysis, all data were normalized using z-score, which subtracts the mean of all observations to each feature and then divides it by its standard deviation. An identification code was assigned to each new HSMAP, in the form of T-HSMAP\#-H, where $\mathrm{T}$ stands for the task (PG, PC, KS); \# stands for the number of the principal component according to the highest explained variance/inertia, and $\mathbf{H}$ stands for hand (dominant-D, non-dominant-ND).

Prior to interpretation, varimax rotation was applied. Next, contributions of the rotated components were analyzed. For each component, the squared loadings were calculated. Following the heuristic method described in [17], interpretation of the component was based primarily on those variables whose contribution is equal to or greater than the average contribution of all variables. Remaining loadings constitute the final HSMAP. For each loading, two items were sought: (1) whether the loading contributes positively or negatively to the variable; (2) whether the loading is associated with a MAP presenting, in itself, construct validity as defined in the exploratory analysis. These two items were used to hypothesize an interpretation and to nominate the HSMAP. Given the subjective nature of said interpretation, the underlying hypotheses as to their meaning will be postulated in the "Discussion" section rather than as part of the results. PCA was carried out in MATLAB® R2016b using the Statistics and Machine Learning Toolbox. 
Table 2 PG MAP correlation

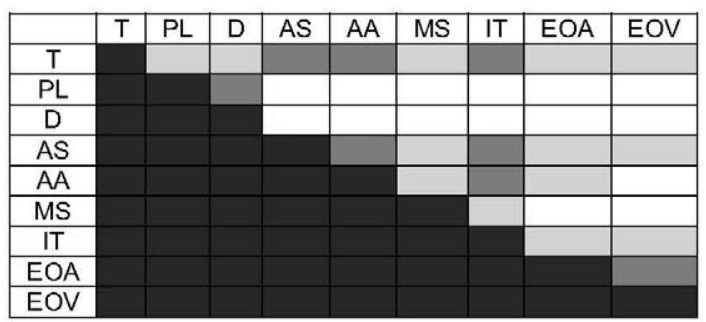

Light shade: $0.4<\rho<0.7$. Dark shade: $\rho>0.7$

\section{Construct validation}

For each HSMAP, statistical significant differences were sought for each cut-off experience threshold established in "Data collection" section. Once again, Mann-Whitney test was employed to look for statistical significant differences between categories $(p<0.05)$. Box plots were calculated in order to get a visual interpretation on the meaning of the HSMAPs, providing information on whether their values increase/decrease with experience, and used to further postulate their meaning. Statistical analysis was once more performed with SPSS v19.

\section{Results}

\section{Data collection}

Twenty-five participants enrolled in the study, of which 11 had a laparoscopic experience $<10$ surgeries (non-Ex10) and $14>10$ surgeries (Ex10). Of the latter, 8 had a laparoscopic experience $>50$ surgeries $($ Ex 50). The remaining 17 were considered as non-Ex 50. All participants had OR experience in laparoscopic surgery, although four had never attended an intervention as main surgeon. All participants completed the PG and PC tasks. Three participants did not carry out the KS task due to scheduling constraints. These included one surgeon in group non-Ex50; and two surgeons in group non-Ex 10 .

\section{MAP exploratory analysis}

Correlation analysis results are shown in Tables 2, 3, 4, 5, and 6. Visual inspection reveals dependencies that justify the application of PCA to the MAPs. PG presents 8 pairs of MAPs correlated with $\rho>0.7$ and 13 with $0.4<\rho<0.7$. PC for the dominant hand presents 12 pairs of MAPs correlated with $\rho>0.7$ and 14 with $0.4<\rho<0.7$. For the nondominant hand, there are 13 and 12 pairs for $\rho>0.7$ and $0.4<\rho<0.7$, respectively. Finally, KS presents 16 pairs of
Table 3 PC MAP correlation (dominant)

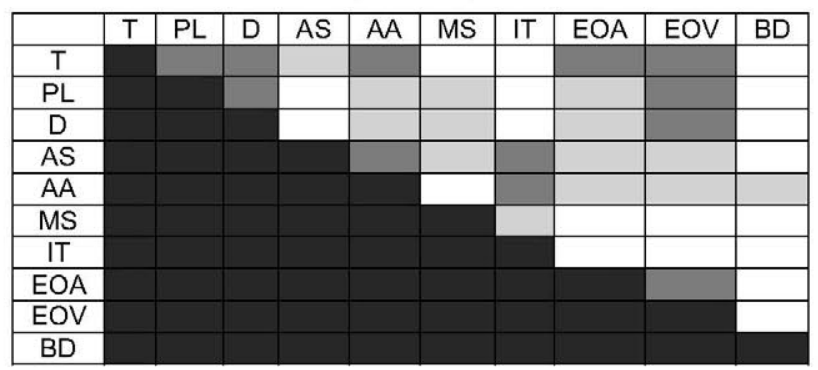

Light shade: $0.4<\rho<0.7$. Dark shade: $\rho>0.7$

Table 4 PC MAP correlation (non-dominant)

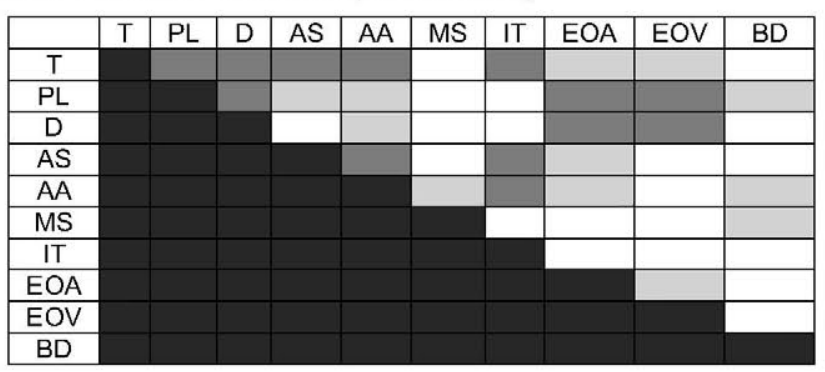

Light shade: $0.4<\rho<0.7$. Dark shade: $\rho>0.7$

Table 5 KS MAP correlation (dominant)

\begin{tabular}{|c|c|c|c|c|c|c|c|c|c|c|}
\hline & T & PL & D & AS & AA & MS & IT & EOA & EOV & BD \\
\hline T & & & & & & & & & & \\
\hline PL & & & & & & & & & \\
\hline D & & & & & & & & & \\
\hline AS & & & & & & & & & \\
\hline AA & & & & & & & & & \\
\hline MS & & & & & & & & & \\
\hline IT & & & & & & & & & \\
\hline EOA & & & & & & & & & \\
\hline EOV & & & & & & & & & \\
\hline BD & & & & & & & & & \\
\hline
\end{tabular}

Light shade: $0.4<\rho<0.7$. Dark shade: $\rho>0.7$

Table 6 KS MAP correlation (non-dominant)

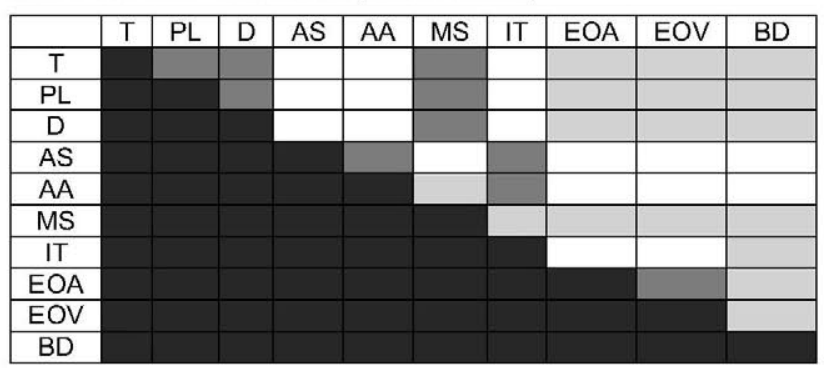

Light shade: $0.4<\rho<0.7$. Dark shade: $\rho>0.7$

MAPs correlated with $\rho>0.7$ and 14 with $0.4<\rho<0.7$ for the dominant hand, and 10 pairs for $\rho>0.7$ and 17 with $0.4<\rho<0.7$ for the non-dominant hand.

Results of the exploratory analysis are shown in Table 7. Overall, the PG task presented more significant differences 
Table 7 MAP exploratory analysis

\begin{tabular}{|c|c|c|c|c|c|c|}
\hline \multirow[t]{2}{*}{ MAPs } & \multicolumn{3}{|c|}{ Ex10-nonEx10 } & \multicolumn{3}{|c|}{ Ex50-nonEx50 } \\
\hline & 1.PG & 2.PC & 3.KS & 1.PG & 2.PC & 3.KS \\
\hline Time & $x$ & $x$ & $x$ & & $\mathrm{x}$ & $x$ \\
\hline Bimanual dexterity & & & $x$ & & $x$ & \\
\hline Dominant hand & 1.PG & 2.PC & 3.KS & 1.PG & 2.PC & 3.KS \\
\hline Path length & & $\mathrm{x}$ & & & $\mathrm{x}$ & $\mathrm{x}$ \\
\hline Depth & & $x$ & & & $x$ & $x$ \\
\hline Av. Speed & $x$ & & & & $x$ & \\
\hline Av. Acceleration & $x$ & & & & $x$ & $x$ \\
\hline Motion smoothness & $x$ & & & & & \\
\hline \multicolumn{7}{|l|}{ Idle time } \\
\hline Economy of area & & & & $\mathrm{x}$ & $x$ & $\mathrm{x}$ \\
\hline Economy of volume & & & & $x$ & $\mathrm{x}$ & $\mathrm{x}$ \\
\hline Non-dominant hand & - & 2.PC & $3 . \mathrm{KS}$ & - & 2.PC & 3.KS \\
\hline Path length & & & $\mathrm{x}$ & & $\mathrm{x}$ & $\mathrm{x}$ \\
\hline Depth & & & $x$ & & $x$ & $x$ \\
\hline Av. Speed & & $x$ & & & $x$ & \\
\hline Av. Acceleration & & $x$ & & & $x$ & \\
\hline \multicolumn{7}{|l|}{ Motion smoothness } \\
\hline Idle time & & & & & $x$ & \\
\hline Economy of area & & & & & & $x$ \\
\hline Economy of volume & & & & & & $x$ \\
\hline
\end{tabular}

Significant differences per task between experience groups are marked with an x. Grey boxes indicate that no MAP is defined for PG non-dominant

between Ex10 and non-Ex10 surgeons. On the other hand, $\mathrm{PC}$ and $\mathrm{KS}$ presented more significant differences when distinguishing between Ex50 and non-Ex50 surgeons. Differences in performance for the non-dominant hand were common at both cut-off thresholds. In general, total time reflected significant differences for all tasks and cut-offs (except for PG in Ex50).

For PG, differences in performance between Ex10 and non-Ex10 surgeons were found mainly with MAPs related to time, kinematic efficiency (av. speed, av. acceleration), and motion smoothness. On the other hand, differences between Ex50 and non-Ex50 surgeons were found related to the spatial efficiency inside the box trainer (economy of area, economy of volume).

For PC, differences in performance between Ex10 and non-Ex10 surgeons were found for MAPs related with time, displacement (path length, depth) of the dominant hand, and kinematic efficiency (av. speed and av. acceleration) for the non-dominant. Most MAPs presented significant differences between Ex50 and non-Ex50 surgeons, with the exception of motion smoothness (both hands), idle time for the dominant hand and economy of area, economy of volume for the nondominant hand.

For KS, differences in performance between Ex10 and non-Ex10 surgeons were found only for time, bimanual experience, path length, and depth of the non-dominant hand. When analyzing differences between Ex50 and nonEx50 surgeons, it was found that neither av. speed, bimanual dexterity, motion smoothness nor idle time showed significant differences for neither hand. The rest of MAPs did present significant differences (except av. acceleration for the non-dominant hand).

\section{PCA for definition of HSMAPs}

Results of applying PCA to the dataset are presented in Table 8. General trends can be observed across the three tasks to reinforce the physical coherence of the contributions. Clusters of MAPs tend to repeat, such as \{path length, depth perception $\},\{$ av. speed, av. acceleration, idle time $\}$ or \{economy of area, economy of volume\}. When contributing to the same HSMAP, time presents always an opposite sign to av. speed and av. acceleration, but the same as path length and depth. Av. speed and av. acceleration always feature opposite sign to idle time. Likewise, path length/depth perception has opposite contributions to economy of area/ economy of volume when both are featured.

Task PG presents three principal components, defining three distinct HSMAPs. PG-HSMAP1-D explains 55.5\% of the inertia, and is contributed mainly by time and kinematic MAPs such as av. speed, av. acceleration and motion smoothness (which individually presented construct validity for Ex10). PG-HSMAP2-D is contributed by path length, depth perception and motion smoothness, and PGHSMAP3-D by economy of area/economy of volume, MAPs that presented construct validity for Ex 50 . 
Table 8 PCA analysis

\begin{tabular}{|c|c|c|c|c|c|c|c|c|c|c|c|}
\hline & Time & \begin{tabular}{|c|} 
Path \\
Length
\end{tabular} & Depth & \begin{tabular}{|c|} 
Av. \\
Speed
\end{tabular} & $\begin{array}{c}\text { Av. } \\
\text { Acceleration }\end{array}$ & $\begin{array}{c}\text { Motion } \\
\text { Smoothness }\end{array}$ & \begin{tabular}{|l|} 
Idle \\
Time
\end{tabular} & $\begin{array}{c}\text { Economy } \\
\text { of Area }\end{array}$ & $\begin{array}{l}\text { Economy } \\
\text { of Volume }\end{array}$ & \begin{tabular}{|l|} 
Bimanual \\
Dexterity
\end{tabular} & $\begin{array}{c}\text { Inertia } \\
(\%)\end{array}$ \\
\hline \multicolumn{12}{|c|}{ - } \\
\hline HSMAP1-D & $\mathbf{x}$ & & & $\mathrm{x}$ & $x$ & $\mathrm{x}$ & & & & & 55.5 \\
\hline HSMAP2-D & & & & & & & & & & & 22.4 \\
\hline HSMAP3-D & & & & & & & & * & * & & 15.5 \\
\hline \multicolumn{12}{|l|}{ PC } \\
\hline HSMAP1-D & $\mathbf{x}^{\star}$ & $\overline{\mathbf{x}^{*}}$ & $\overline{\mathbf{x}^{*}}$ & & & & & * & * & & 54.4 \\
\hline HSMAP2- D & & & & * & * & & & & & & 26.3 \\
\hline HSMAP3- D & & & & & & & & & & * & 9.1 \\
\hline HSMAP1- ND & & * & * & & & & & & & & 53.3 \\
\hline HSMAP2- ND & $x^{*}$ & & & $\mathbf{x}^{*}$ & $\mathbf{x}^{*}$ & & * & & & & 21.5 \\
\hline HSMAP3-ND & & & & & & & & & & * & 12.3 \\
\hline \multicolumn{12}{|l|}{ KS } \\
\hline HSMAP1-D & & * & * & & * & & & & & & 65.4 \\
\hline HSMAP2- D & $\mathbf{x}^{*}$ & & & & & & & * & * & & 20.7 \\
\hline HSMAP1- ND & $\mathbf{x}^{\star}$ & $\mathbf{x}^{\star}$ & $\mathbf{x}^{\star}$ & & & & & * & * & & 52.9 \\
\hline HSMAP2- ND & & & & & & & & & & & 29.6 \\
\hline
\end{tabular}

For each task, the eigenvalue composition of each HSMAP is color-coded. Shadowed boxes reflect a significant contribution of the MAP to the HSMAP. Grey shadows: positive contributions; black shadows: negative contributions. x: MAPs with Ex10 construct validity. *: MAPs with Ex 50 construct validity. The last column reflects the $\%$ of the total inertia/variance explained by the component

Task PC presents three HSMAPs per hand. Even though the inertia of the first two components for the dominant hand explain slightly above $80 \%$ of the variance, we decided to include a third component so as to maintain the same number of components between both hands in the search of possible symmetries. Contributions to both hands are indeed nearly symmetrical, with the exception of time, which contributes positively to PC-HSMAP1-D and negatively to PC-HSMAP2-ND. For both hands, PC-HSMAP1 are positively contributed by path length and depth perception, and negatively by economy of area/economy of volume. These HSMAPs help explain $54.4 / 53.3 \%$ of the variance for the dominant/non-dominant hand, respectively. PC-HSMAP2 are positively related to av. speed, av. acceleration and negatively to idle time. Finally, a $9.1 / 12.3 \%$ of variance is explained by PC-HSMAP3 (respectively, for the dominant and non-dominant hand), which account for motion smoothness and bimanual dexterity.

Task KT presents two HSMAPs per hand. Unlike PC, there are several differences between the dominant and non-dominant hand. KS-HSMAP1-D is positively related with path length, depth perception, av. speed, av. acceleration and motion smoothness, and negatively with idle time. This HSMAP explains $65.4 \%$ of the inertia. KSHSMAP2-D is contributed positively by economy of area and economy of volume and negatively by time, and explains $20.7 \%$ of the inertia. KS-HSMAP1-ND is contributed positively by time, path length, depth perception and motion smoothness, and negatively by economy of area/economy of volume. Explained variance is $52.9 \%$. KS-HSMAP2-ND explains a $29.6 \%$ of variance, and is contributed positively by av. speed, av. acceleration, and negatively by idle time.

\section{Construct validation}

Box plot scores' distributions by cut-off thresholds are shown in Fig. 2, while results of the construct validation analysis for the HSMAPs are presented in Table 9. Task PG presents construct validation for PG-HSMAP1-D and PG-HSMAP3-D for the Ex10 and Ex50 thresholds, respectively, reflecting the corresponding construct validation of their contributing individual MAPs. Box plots show that the former tends to be lower with experience, while the latter tends to increase.

Task PC presents construct validation for PC-HSMAP1D and PC-HSMAP2-ND for both cut-off thresholds. Box plot analysis shows that the former tends to decrease with experience, whilst the latter increases. PC-HSMAP1-ND presents construct validation for Ex 50, taking lower values for experienced surgeons. PC-HSMAP2-D presents construct validation for Ex 50, taking higher values for experienced surgeons.

Task KS presents construct validation for KS-HSMAP2D and KS-HSMAP1-ND at both cut-off thresholds, with the first one increasing with experience and the second one decreasing.

\section{Discussion}

In this study, we have analyzed the feasibility of defining combined MAPs for the assessment of MIS technical skills using PCA. Our goal has been to prove several points: (1) that combinations of MAPs into HSMAPs can provide meaningful performance information which can be translated to feedback for the trainee; (2) that said HSMAPs may 
Fig. 2 HSMAP box plots. Blue-HSMAP1; GreenHSMAP2; Brown-HSMAP3. (Color figure online)

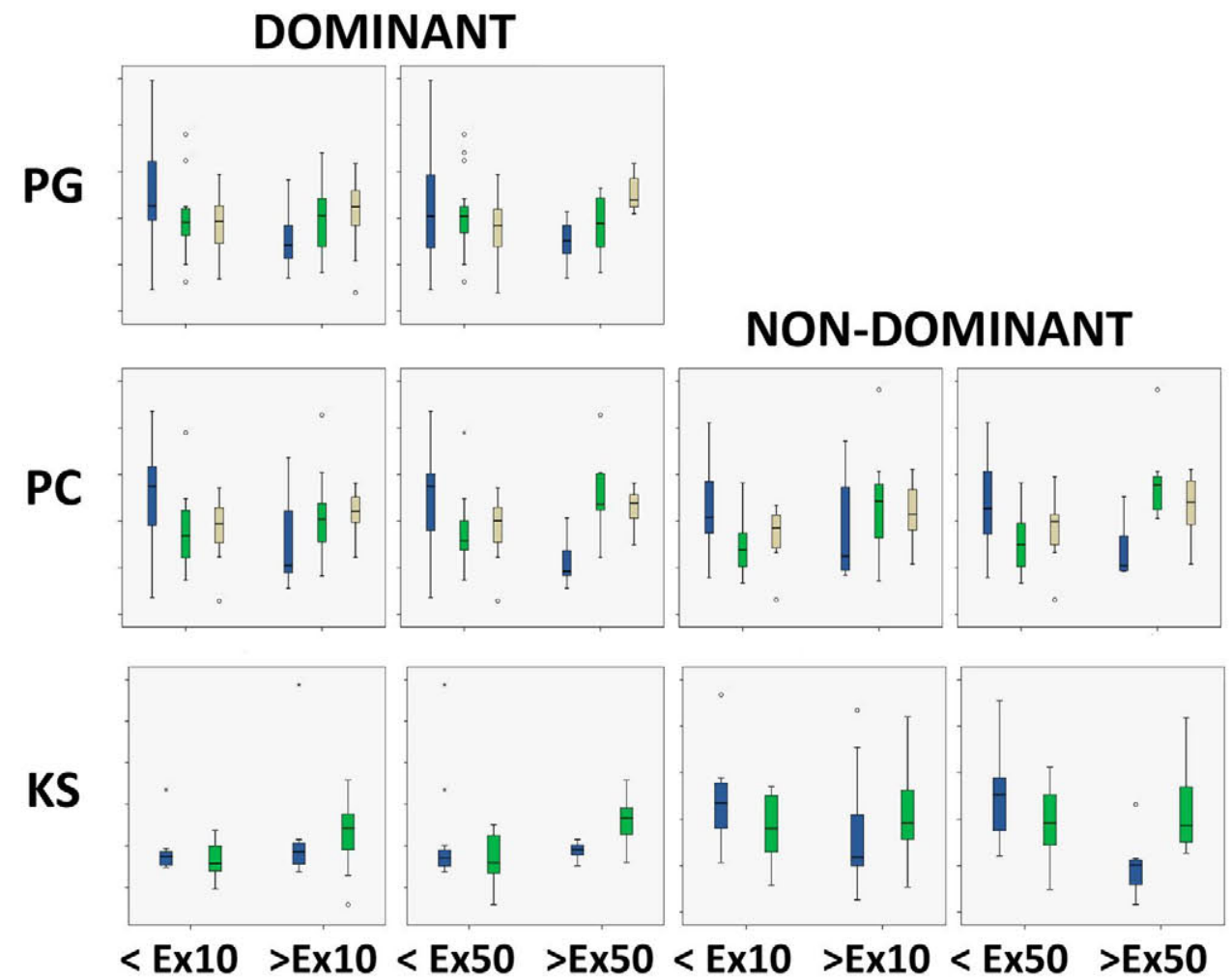

Table 9 HSMAP construct validation

\begin{tabular}{|c|c|c|c|c|c|c|}
\hline HSMAPS & \multicolumn{3}{|c|}{ Ex10-nonEx10 } & \multicolumn{3}{|c|}{ Ex50-nonEx50 } \\
\hline Dominant hand & 1.PG & 2.PC & 3.KS & 1.PG & 2.PC & 3.KS \\
\hline HSMAP1 & $x$ & $x$ & & & $\mathrm{x}$ & \\
\hline HSMAP2 & & & $\mathrm{x}$ & & $x$ & $\mathrm{x}$ \\
\hline HSMAP3 & & & & $x$ & & \\
\hline Non-dominant hand & - & 2.PC & 3.KS & - & 2.PC & 3.KS \\
\hline HSMAP1 & & & $x$ & & $x$ & $x$ \\
\hline HSMAP2 & & $x$ & & & $x$ & \\
\hline HSMAP3 & & & & & & \\
\hline
\end{tabular}

Significant differences per task between experience groups are marked with an x. Numbers of HSMAPs refer to each task's corresponding HSMAP as presented in Table 8. Grey boxes indicate that no HSMAP is defined for that combination of task and hand

have sufficient predictive power to discriminate between experience levels, always taking into account the nature and difficulty of the task at hand; and (3) that it is not possible to generalize the use of metrics (and even HSMAPs) to all box trainer tasks, as the different nuances in a task's learning goals have an influence on the prevalence of one MAP vs. another one (e.g., a MAP such as path length may not have the same pedagogical weight in a cutting task, where movements are more localized, than on a peg grasping task).

Up to this point, the study has focused on presenting the raw, objective data derived from PCA. However, our final aim is, starting from our set of identified HSMAPs, to ascertain some significant, didactic interpretation from them. It is therefore necessary at this point to postulate certain hypotheses based on the results here presented, for each task and HSMAP. These will need to be ratified or challenged in future studies.

Task PG was initially considered to be the easiest task by the authors, since it does not involve bimanual coordination. An implementation of the PG task had already been validated using the EVA tracking system in [4]. Three HSMAPs were calculated, covering most of the variance of results. The dependencies of PG-HSMAP1-D with time, av. speed, av. acceleration, motion smoothness, and idle time lead us to induce that the parameter is strongly related with confidence of movements. Since the parameter decreases with experience, we can rename it as "Insecurity of movements." From the authors' experience, this is coherent, as non-experienced surgeons tend to show low depth perception, which translates to jerkier movements and longer completion times. 
PG-HSMAP2-D is contributed by path length, depth, and motion smoothness, which, following other studies in the field [7], could be understood as "Economy of movements," or how to achieve a goal with the least displacement possible. Finally, PG-HSMAP3-D is clearly related with spatial efficiency, and thus we can rename it as "Economy of space."

Task PC had previously undergone validation in [5] using the EndoVis augmented box trainer. In the present study, three HSMAPs were identified per hand. At a first glance, dominant and non-dominant hand parameters share a similar composition of MAPs. However, one must also take into account the role of each hand in the task. The dominant hand performs the actual pattern cutting, while the nondominant hand keeps the tissue tight to make cutting possible. Looking at Table 8, one can see that PC-HSMAP1-D requires dominion of the space, the trajectory followed and the depth of the instruments. Figure 2 shows a decrease of its value with experience. Thus, we can interpret this HSMAP as "Cutting efficacy." Likewise, and given the supporting role of PC-HSMAP1-ND, and the fact that attention tends to be focused on the main hand, we interpret its meaning as "Peripheral unawareness." This is due to the fact that experienced surgeons will move less the non-dominant hand and keep it less in focus while their attention is placed on the dominant hand. Another possible interpretation could be in terms of "Tissue exposure insecurity," since the role of this hand is to tract the tissue into place to enable cutting with the dominant hand. First-hand experience of authors suggests that these movements tend to be smaller and more controlled, which is reflected by trajectory and spatial efficiency MAP contributions. PC-HSMAP2-D is directly related with av. speed and av. acceleration, inversely with idle time, and tends to increase with experience. We may interpret it as "Confidence of dominant movements." PC-HSMAP2-ND shows similar contributions; therefore, we can relate this parameter with "Confidence of non-dominant movements." Finally, the third HSMAP (for both hands) is closely contributed by motion smoothness and bimanual dexterity. This could be interpreted separately, as "Motion smoothness" for each hand, or used to build a compound HSMAP averaging both values, to reflect "Bimanual dexterity."

Task KS had also undergone previous validation in [5] using the EndoVis augmented box trainer. In this task, the degree of coordination must be high to perform efficiently the knot, and thus both hands assume a major role in the action. In our study, we found that two HSMAPs per hand can cope with $>90 \%$ of the variance. A limitation of our study is that it does not distinguish between the different phases of the task (needle driving and knotting), making difficult the interpretation of the HSMAPs. Thus, KSHSMAP1-D is contributed by displacement and kinematic MAPs, with no clear distinction between experienced and non-experienced surgeons and no construct validity. Tentatively, we name it "Needle driving precision," considering that the most precise movements are performed during puncturing and needle driving of the tissue. KS-HSMAP2-D clearly increases with experience, and considering its contributions could be related with "Economy of space." KSHSMAP1-ND decreases with experience, and is contributed by time, path length, depth, motion smoothness, economy of area, and economy of volume. Again, considering that the most precise motions must be carried out during knotting, we could establish this HSMAP as "Knotting insecurity." Finally, KS-HSMAP2-ND is related with av. speed, av. acceleration and idle time, with no clear differences between experienced and non-experienced surgeons. As in the other tasks, we could tentatively associate it with "Confidence of movements."

Table 10 sums the hypotheses above covered. If one is to interpret them in light of the construct validation results, then in hand-eye coordination/grasping tasks, the first skill (measurable by motion analysis) to be honed involves confidence of movements; whilst further experience helps to refine the perception of the working space. Cutting tasks show that experience progressively makes one perform more precise dissections, while at the same time master the awareness of the non-dominant hand. At higher levels of experience, movements of the non-dominant hand tend to be more efficient as the peripheral attention on said hand is mastered. Finally, suturing dominion involves a progressive increase on the efficient use of the available workspace with the dominant hand, while at the same time performing more precise, coordinated and smoother movements with the nondominant hand.

Naturally, one must take these interpretations with care, and consider the limitations of the study. To begin with, the bias of the authors need to be taken into account, since as mentioned earlier, interpretations are based on their own experience, whether it should be clinical or technological. Moreover, these interpretations can suffer bias from only considering motion analysis information. While this was the goal set for this study (to infer a higher meaning from the use of MAPs), there are nuances and actions that cannot be alone considered with this approach. Data on errors, instruments off-camera, etc. provide a valuable information that current motion analysis systems are not able to measure. Similarly, the choice of MAPs alone influences the configuration of the HSMAPs. If one where to choose another set of MAPs, results could differ from those obtained here. New HSMAP configurations, whether based only on MAPs or considering qualitative information, could likely benefit from the methodology here presented. The methodological choices behind PCA could also influence the results if, for example, one were to choose another approach to this multivariate analysis, or set a different stopping condition when choosing the 
Table 10 HSMAP

interpretation hypotheses

\begin{tabular}{|c|c|c|c|c|}
\hline \multirow[t]{2}{*}{ HSMAP } & \multirow[t]{2}{*}{$\begin{array}{l}\text { Evolution with } \\
\text { experience }\end{array}$} & \multirow[t]{2}{*}{ Interpretation } & \multicolumn{2}{|c|}{$\begin{array}{l}\text { Construct } \\
\text { validation }\end{array}$} \\
\hline & & & $\operatorname{Ex} 10$ & Ex50 \\
\hline \multicolumn{5}{|l|}{ PG } \\
\hline PG-HSMAP1-D & Decrease & Insecurity of movements & $\mathrm{x}$ & \\
\hline PG-HSMAP2-D & - & Economy of movements & & \\
\hline PG-HSMAP3-D & Increase & Economy of space & & $\mathrm{x}$ \\
\hline \multicolumn{5}{|l|}{$\mathrm{PC}$} \\
\hline PC-HSMAP1-D & Decrease & Cutting efficacy & $\mathrm{x}$ & $\mathrm{x}$ \\
\hline PC-HSMAP2-D & Increase & Confidence of dominant movements & & $\mathrm{x}$ \\
\hline PC-HSMAP3-D & - & Bimanual dexterity/motion smoothness & & \\
\hline PC-HSMAP1-ND & Decrease & Peripheral unawareness/tissue exposure insecurity & & $\mathrm{x}$ \\
\hline PC-HSMAP2-ND & Increase & Confidence of non-dominant movements & $\mathrm{x}$ & $\mathrm{x}$ \\
\hline PC-HSMAP3-ND & - & Bimanual dexterity/motion smoothness & & \\
\hline \multicolumn{5}{|l|}{ KS } \\
\hline KS-HSMAP1-D & - & Needle driving precision & & \\
\hline KS-HSMAP2-D & Increase & Economy of space & $\mathrm{x}$ & $\mathrm{x}$ \\
\hline KS-HSMAP1-ND & Decrease & Knotting insecurity & $\mathrm{x}$ & $\mathrm{x}$ \\
\hline KS-HSMAP2-ND & - & Confidence of non-dominant movements & & \\
\hline
\end{tabular}

For evolution with experience, only those HSMAPs presenting construct validity and a clear representation in the box plots of Fig. 2 are considered number of principal components. Another major limitation is the number of participants in the study. In order to infer more significant statistical conclusions to our results, new studies should be carried out with a higher number of volunteers with different experiences. Finally, all volunteers were right handed, and thus we cannot generalize to left-handed residents without further proof.

These factors should be reason enough to encourage further research in this field. New validation studies should be performed in order to prove the real pedagogical value of the new HSMAPs. These studies should explore not only the validity of the HSMAPs, but their underlying pedagogical value. For our part, our next steps include the following: (1) further validation studies with new recruits performing the tasks (or similar versions) and recorded with the EVA tracking system; (2) an analysis of the video recordings obtained, carried out by independent expert evaluators. With respect to the latter point, we intend to use the GOALS scale as a comparative scoring system, in order to follow up the work presented in [1]. More to the point, GOALS items present certain a priori similitudes with the interpretation of our HSMAPs, evaluating depth perception, efficiency, bimanual dexterity, and tissue handling (partially related with motion smoothness) [10].

Without disregarding the challenges ahead, we remain confident that the interpretation of motion analysis in light of its pedagogical value should be further explored at this point. The literature shows us that MAPs can be used to distinguish between expert and non-expert surgeons in box trainer tasks. However, there are still discrepancies on the specific MAPs, tasks, and skills that match with each other, reflecting that the use of motion analysis cannot be generalized independently of the expected learning outcomes of a task. A better understanding of the actions supported by the movements of the instruments may provide new insights on this field, increase the pedagogical value of motion analysis and develop new mechanisms to provide meaningful, constructive feedback during training.

\section{Conclusions}

Motion analysis is frequently used as an assessment tool for laparoscopic basic skills' training, both in box trainers and VR simulators. Validation studies have shown that they can provide distinct information on a surgeon's MIS skills in these settings. The challenge, however, remains in analyzing the dependencies between MAPs in order to infer a higher meaning that increases their pedagogical value. In this work, we have presented a methodological approach to this end. This study knows its limitations, and as such does not intend to provide definitive conclusions on the subject. Rather, we intend to stimulate this research path within the surgical training community. We feel that, with such a large body of literature devoted to MIS motion analysis, there is still a gap to be explored with respect to its impact and correlation with MIS skills such as tissue handling or knotting. A successful end to this road could result in new potential services 
and applications to improve surgical training and attract the interest of stakeholders in the field, from the provision of constructive feedback mechanisms to the development of new decision support systems able to predict a trainee's level of MIS technical skills.

Acknowledgements The authors would like to acknowledge all staff of the Jesús Usón Minimally Invasive Surgery Centre involved in setting up and performing the experiments described in this work.

\section{Compliance with ethical standards}

Disclosures Drs. I. Oropesa, F. Pérez Escamirosa, J.A. Sánchez Margallo, S. Enciso, B. Rodríguez-Vila, A. Minor Martínez, F.M SánchezMargallo, P. Sánchez-González, and E.J. Gómez have no conflict of interests or financial ties to disclose.

\section{References}

1. Sánchez-Margallo JA, Sánchez-Margallo FM, Oropesa I, Enciso S, Gómez EJ (2017) Objective assessment based on motionrelated metrics and technical performance in laparoscopic suturing. Int J Comput Assist Radiol Surg 12:307-314. https://doi. org/10.1007/s11548-016-1459-3

2. van Hove PD, Tuijthof GJM, Verdaasdonk EGG, Stassen LPS, Dankelman J (2010) Objective assessment of technical surgical skills. Br J Surg 97:972-987. https://doi.org/10.1002/bjs.7115

3. Partridge RW, Brown FS, Brennan PM, Hennessey IAM, Hughes MA (2016) The LEAP ${ }^{\text {TM }}$ gesture interface device and takehome laparoscopic simulators. Surg Innov 23:70-77. https://doi. org/10.1177/1553350615594734

4. Oropesa I, Sánchez-González P, Chmarra MK, Lamata P, Fernández Á, Sánchez-Margallo JA, Jansen FW, Dankelman J, SánchezMargallo FM, Gómez EJ (2013) EVA: laparoscopic instrument tracking based on endoscopic video analysis for psychomotor skills assessment. Surg Endosc 27:1029-1039. https://doi. org/10.1007/s00464-012-2513-z

5. Escamirosa FP, Flores RMO, Oropesa I, Vidal CRZ, Martínez AM (2015) Face, content, and construct validity of the EndoViS training system for objective assessment of psychomotor skills of laparoscopic surgeons. Surg Endosc 29:3392-3403. https://doi. org/10.1007/s00464-014-4032-6

6. Hofstad EF, Våpenstad C, Chmarra MK, Langø T, Kuhry E, Mårvik R (2013) A study of psychomotor skills in minimally invasive surgery: what differentiates expert and nonexpert performance. Surg Endosc 27:854-863. https://doi.org/10.1007/ s00464-012-2524-9

7. Oropesa I, Sánchez-González P, Lamata P, Chmarra MK, Pagador JB, Sánchez-Margallo JA, Sánchez-Margallo FM, Gómez EJ (2011) Methods and tools for objective assessment of psychomotor skills in laparoscopic surgery. J Surg Res 171:e81-e95. https:// doi.org/10.1016/j.jss.2011.06.034

8. Feldman LS, Sherman V, Fried GM (2004) Using simulators to assess laparoscopic competence: ready for widespread use? Surgery 135:28-42. https://doi.org/10.1016/S0039-6060(03)00155-7

9. Fried GM, Feldman LS (2008) Objective assessment of technical performance. World J Surg 32:156-160. https://doi.org/10.1007/ s00268-007-9143-y

10. Vassiliou MC, Feldman LS, Andrew CG, Bergman S, Leffondré K, Stanbridge D, Fried GM (2005) A global assessment tool for evaluation of intraoperative laparoscopic skills. Am J Surg 190:107-113. https://doi.org/10.1016/j.amjsurg.2005.04.004

11. Chang J, Banaszek DC, Gambrel J, Bardana D (2016) Global rating scales and motion analysis are valid proficiency metrics in virtual and benchtop knee arthroscopy simulators. Clin Orthop Relat Res 474:956-964. https://doi.org/10.1007/s11999-015-4510-8

12. Cristancho SM, Hodgson AJ, Panton ONM, Meneghetti A, Warnock G, Qayumi K (2009) Intraoperative monitoring of laparoscopic skill development based on quantitative measures. Surg Endose 23:2181-2190. https://doi.org/10.1007/ s00464-008-0246-9

13. Chmarra MK, Klein S, De Winter JCF, Jansen FW, Dankelman $\mathrm{J}$ (2010) Objective classification of residents based on their psychomotor laparoscopic skills. Surg Endosc 24:1031-1039. https:// doi.org/10.1007/s00464-009-0721-y

14. Oropesa I, Sánchez-González P, Chmarra MK, Lamata P, PérezRodríguez R, Jansen FW, Dankelman J, Gómez EJ (2013) Supervised classification of psychomotor competence in minimally invasive surgery based on instruments motion analysis. Surg Endosc 28:657-670. https://doi.org/10.1007/s00464-013-3226-7

15. Horeman T, Dankelman J, Jansen FW, van den Dobbelsteen JJ (2014) Assessment of laparoscopic skills based on force and motion parameters. IEEE Trans Biomed Eng 61:805-813. https:// doi.org/10.1109/TBME.2013.2290052

16. Enciso Sanz S, Sánchez Margallo FM, Díaz-Güemes MartínPortugués I, Usón Gargallo J (2012) Preliminary validation of the Simulap(B) physical simulator and its assessment system for laparoscopic surgery. Cir Esp 90:38-44. https://doi.org/10.1016/j. ciresp.2011.07.013

17. Abdi HE, Williams LJ (2010) Principal component analysis. WIREs Comp Stat 2:433-459. https://doi.org/10.1002/wics.101 